\title{
Compositional influence on the electrical performance of zinc indium tin oxide transparent thin-film transistors
}

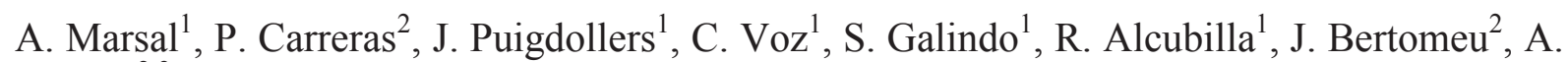 \\ Antony ${ }^{2,3}$
}

1 Dept Enginyeria Electronica and Center of Research in Nanoengineering, Universitat Politècnica Catalunya. Barcelona (Spain)

2 Dept Física Aplicada i Òptica, Universitat de Barcelona. Barcelona (Spain)

3 Indian Institute of Technology, Bombay (India)

Keywords: Zinc-Indium-Tin-Oxide; Thin Films; Thin-Film Transistor; Density-of-States

\begin{abstract}
In this work, zinc indium tin oxide layers with different compositions are used as the active layer of thin film transistors. This multicomponent transparent conductive oxide is gaining great interest due to its reduced content of the scarce indium element. Experimental data indicate that the incorporation of zinc promotes the creation of oxygen vacancies. In thin-film transistors this effect leads to a higher threshold voltage values. The field-effect mobility is also strongly degraded, probably due to coulomb scattering by ionized defects. A post deposition annealing in air reduces the density of oxygen vacancies and improves the fieldeffect mobility by orders of magnitude. Finally, the electrical characteristics of the fabricated thin-film transistors have been analyzed to estimate the density of states in the gap of the active layers. These measurements reveal a clear peak located at $0.3 \mathrm{eV}$ from the conduction band edge that could be attributed to oxygen vacancies.
\end{abstract}

\section{Introduction}

Transparent conductive oxides (TCOs) have attracted much attention in the optoelectronic industry during the last years due to its emerging interest in transparent electronics [1, 2]. TCOs are degenerately doped semiconductors with a band gap higher than $3 \mathrm{eV}$ and thus, conductive and transparent in the visible wavelength range. These properties, combined with the possibility of processing layers at low temperatures, made them suitable for several applications such as solar cells or flexible electronic devices. It is important to study and characterize the films, to further understand the interaction of each layer within the device.

Among all the TCOs, indium tin oxide (ITO) is widely used because of its low resistivity (in the order of $10^{-4} \Omega \cdot \mathrm{cm}$ ) and high transmittance (higher than $80 \%$ ) [1]. However, it has the disadvantage of being expensive due to the scarcity of indium. On the contrary, zinc oxide $(\mathrm{ZnO})$ seems to be a promising material to substitute ITO given its comparable electrical and optical properties and the abundance of $\mathrm{Zn}$ in the Earth's crust. For this reason, quaternary compounds such as zinc indium tin oxide (ZITO) have attracted much interest to lower the concentration of indium [1]. The electrical, optical and morphological properties of this alloy have been previously characterized [3,4]. Moreover, its electronic band structure has been 
also carefully studied [5]. The aim of this work is to study the electronic properties of different ZITO layers by manufacturing thin film transistors (TFTs) [6 - 10].

In the present study, a series of amorphous ZITO thin films with Zn cation concentration ranging from $17 \%$ to $67 \%$, as well as pure ITO and $\mathrm{ZnO}$ thin films were employed as TFT active layers in order to compare their behaviour within the device. As the $\mathrm{Zn}$ concentration increases, the $\mathrm{O}$ vacancies at the layer surface are said to increase $[2,5]$, thus indicating a higher density of charge carriers (electrons in this case). An annealing process in air atmosphere has been applied to each layer in order to reduce the carrier concentration by decreasing the number of $\mathrm{O}$ vacancies. According to methodologies used to describe metaloxide semiconductor field-effect transistors [1], we have extracted parameters such as carrier mobility, drain current on-off ratio and threshold voltage, which are three of the basic parameters to quantify TFTs performance. Besides, the density-of-states (DOS) will be estimated by analyzing the temperature dependence of the channel conductance following a procedure described elsewhere [11].

\section{Experimental details}

All the TFTs with ZITO active channel studied herein have been fabricated using a bottom gate-top contact structure as depicted in figure 1. A $p$-type Si wafer $\left(N_{A} \sim 10^{16} \mathrm{~cm}^{-3}\right)$ with a thermally oxidized layer $\left(250 \mathrm{~nm}\right.$-thick $\left.\mathrm{SiO}_{2}\right)$ has been employed as a substrate and gate insulator, respectively. In this application, p-type wafers are more suitable to replicate a metallic gate electrode because ZITO semiconductors ( $n$-type) operate in accumulation under a positive voltage applied to the gate [8]. $\mathrm{SiO}_{2}$ was etched from the wafer rear-side before further deposition steps were performed.

ZITO samples were deposited over the oxidized front-side of $p$-type $\mathrm{Si}$ wafers at the University of Barcelona by radio frequency (RF) magnetron co-sputtering of $\mathrm{ZnO}$ and ITO $\left(\mathrm{In}_{\mathrm{s}} \mathrm{O}_{3}\right.$ doped with $\left.10 \% \mathrm{SnO}_{2}\right)$ at room temperature. 3-inch target purity was of $99.995 \%$ for $\mathrm{ZnO}$ and $99.99 \%$ for ITO. The equipment used was an ATC ORION sputtering from AJA International, Inc. The process consists of simultaneously sputtering two targets by applying a constant RF power $(50 \mathrm{~W})$ to ITO target and increasing the power applied to $\mathrm{ZnO}$ one from $25 \mathrm{~W}$ to $150 \mathrm{~W}$ in 6 steps of $25 \mathrm{~W}$, thus getting 6 kind of layers ranging from $17 \%$ to $67 \%$ of $\mathrm{Zn}$ content ratio to whole metal content, as determined from X-ray Photoelectron Spectroscopy (XPS) analyses. Samples were kept at $11.7 \mathrm{~cm}$ away from the targets, with a rotation speed of $10 \mathrm{rpm}$. The thickness of the layers ranged between 35 to $50 \mathrm{~nm}$. Each deposition process was performed over 2 samples, having a total of 16 samples with 6 different $\mathrm{Zn}$ contents plus pure ITO and $\mathrm{ZnO}$.

Increasing the $\mathrm{Zn}$ content increases the $\mathrm{O}$ vacancy density at the film surface, as it has been reported by Carreras et al. [5, 12], thus resulting in a highly conductive surface layer [13]. To reduce this effect, one sample of each kind was annealed in air atmosphere. Each sample was heated during $1 \mathrm{~h}$ at $300{ }^{\circ} \mathrm{C}$, with a temperature ramp-up of $30 \mathrm{~min}$. The aim of the thermal treatment was to oxidize the film to decrease the carrier concentration by reducing the oxygen vacancy density [14] or by chemisorption of oxygen at the ZITOs surface and nano-grain boundaries. The morphology of the samples was previously characterized by X-Ray 
Diffraction Spectroscopy and High-Resolution Transmission Electron Microscopy [15]. Results showed that the structure is not completely amorphous as deduced by XRD, but it shows nanocrystals embedded in a compact amorphous structure.

Gold drain and source electrodes were thermally evaporated through a shadow mask at a pressure below $10^{-3} \mathrm{~Pa}$ directly over the ZITO films. The thickness of gold electrodes was 50 $\mathrm{nm}$. Channel width and length were $2.6 \mathrm{~mm}$ and $80 \mu \mathrm{m}$, respectively.

Resistivity measurements were performed by a four point probe system (Jandel RM3). This measurement was performed with the same ZITO layers deposited over a glass substrate. All other electric measurements were performed inside a low-vacuum chamber $(<10 \underline{\mathrm{Pa}})$, in dark conditions using an Agilent 4156C source/monitor. To measure the temperature dependence of the electrical characteristics, all the devices were heated by means of a MMR Technologies temperature controller (model K-20).

\section{Results}

The composition of the films is summarized in table 1 where RF magnetron power, estimated cation content ratios and resistivity are shown. All these data were obtained as described above.

All the TFTs have been electrically characterized according to standard models [16]. The current that flows along the TFT channel is a function of the gate to source $\left(V_{G S}\right)$ and drain to source voltages $\left(V_{D S}\right)$ distinguishing among three operational regimes (subthreshold, linear and saturation). Under saturation regime, in which $V_{D S}$ is greater than $V_{G S}-V_{T}$, the current can be described as follows:

$$
D S=\frac{W \cdot C_{o x} \cdot \mu}{2 L}\left(V_{G S}-V_{T}\right)^{2}
$$

where $W$ and $L$ are the width and length of the channel respectively, $C_{o x}$ is the gate capacitance per unit area, $\mu$ represents the field-effect mobility of carriers along the channel and $V_{T}$ the threshold voltage, designing the minimum voltage at which appreciable current flows.

All the fabricated TFTs have been measured under the same voltage range in order to compare the different active layers. Figure 2 depicts $I_{D S} v s$. $V_{D S}$ (output characteristic) at several $V_{G S}$ voltages ranging from $0 \mathrm{~V}$ to $20 \mathrm{~V}$ for both, annealed and as deposited ZITO50 films. This TFT showed linear regime before the pinch-off condition is achieved $\left(V_{D S} \sim 10 \mathrm{~V}\right.$, for $V_{G S}=20 \mathrm{~V}$ ) and an excellent saturation regime at which current is constant with respect to $V_{D S}$. This device exhibited hard saturation which means that the slope of each $I_{D S}$ curve is practically zero, indicating an constant carrier distribution in the entire thickness of the channel.

Figure 3 illustrates the $I_{D S}-V_{G S}$ behaviour (transfer characteristic) for a ZITO100 TFT with $V_{G S}$ from -2 to $20 \mathrm{~V}$ and a fixed $V_{D S}$ of $20 \mathrm{~V}$. The transfer characteristics with and without annealing treatment are depicted. A $10^{6}$ on-to-off current ratio $\left(I_{o n} / I_{o f f}\right)$ can be observed in the 
annealed films (between $5 \mathrm{~V}$ and $20 \mathrm{~V}$ with a maximum current of $13 \mu \mathrm{A}$ and a minimum of $9.5 \mathrm{pA}$ ) while a lower ratio of $10^{3}$ is found for the other device.

To extract the mobility and threshold voltage, transfer measurements were performed under saturation regime (figure 4); $V_{G S}$ was swept from $-2 \mathrm{~V}$ to $15 \mathrm{~V}$ and $V_{D S}$ was fixed at $20 \mathrm{~V}$. The fit of the saturation characteristic allows us to obtain the field-effect mobility and the threshold voltage as a function of $\mathrm{Zn}$ concentration (figure 5). This graph shows for both, asdeposited and annealed TFTs, an initial rise of the threshold voltage to positive values and a later stabilization as the $\mathrm{Zn}$ concentration increases. The evolution of both curves is similar although there is a higher threshold voltage difference at the lowest $\mathrm{Zn}$ concentration. On the other hand, the field-effect mobility shows important changes not only due to the Zn content but also to the annealing treatment. The TFTs with annealed ZITO films suffer a noticeable mobility drop of three orders of magnitude, while non-annealed devices undergo an up to five orders of magnitude reduction. It should be noted that samples with 54, 63 and $67 \%$ of $\mathrm{Zn}$ content presented the highest drop in field-effect mobility.

To study the DOS of ZITO films, transfer characteristics were measured at different temperatures ranging from $300 \mathrm{~K}$ to $370 \mathrm{~K}$ in steps of $10 \mathrm{~K}$. Figure 6 depicts a current increase in a ZITO100-channel TFT as temperature rises. An Arrhenius plot of the channel current (not represented herein) evidenced a thermally activated behaviour $\sigma=\sigma_{0} \exp \left(-E_{a} /\right.$ $K T)$, where the activation energy $\left(E_{a}\right)$ can be interpreted as the energy barrier for thermal release of trapped carriers, thus associated to the difference between the Fermi level position $\left(E_{F}\right)$ and conduction band $\left(E_{C B}\right)$. The inset in figure 6 shows the extracted $E_{a}$ from the Arrhenius plot of the measured thermal evolution, as a function of the gate voltage. The rate at which $E_{a}$ varies with respect to $V_{G S}$ indicates how the Fermi level can shift through the DOS distribution in the band gap. A fast variation of $E_{F}$ towards the conduction band would represent a low density of localized states to be filled by carriers and vice versa. The DOS in the gap of the semiconductor can be calculated from the derivative of $E_{a}$ with respect to $V_{G S}$ [17].

$$
\operatorname{DOS}(E)=\frac{C_{o x}}{q} \frac{1}{t\left(d E_{a} / d V_{G S}\right)}
$$

Where $E$ is the energy measured from the conduction band edge and $t$ the thickness of the accumulation channel.

\section{Discussion}

Focusing firstly on not annealed TFTs, it can be appreciated (table 1) a resistivity increase with increasing $\mathrm{Zn}$ content. Resistivity is inversely proportional to both, carrier mobility and carrier density, which may bring us to conclude that TFTs with higher Zn content have a lower mobility or less free carriers [18]. The initial carrier density $\left(n_{0}\right)$ is directly proportional to the threshold voltage which can be approached [1] according to:

$$
V_{T}=V_{F B}-\frac{q \cdot d \cdot n_{0}}{C_{o x}}
$$


where $V_{F B}$ is the flat-band voltage, $q$ is the electronic charge and $d$ is the oxide thickness. In fact, threshold voltage starts from negative values for low $\mathrm{Zn}$ concentration, meaning that these films present a high carrier concentration [12] as appointed also by resistivity measurements.

On the other hand, field-effect mobility drops dramatically (down to $10^{-5}$ ) when increasing the $\mathrm{Zn}$ content. This result is an agreement with the reported by Ryu et al. [9] The decrease of the mobility values is in good agreement with a supposed rise of oxygen vacancies with an increasing $\mathrm{Zn}$ content ratio [2, 5]: a higher oxygen vacancy density enhances the coulomb scattering of free electrons by the ionized defects, thus causing the degradation of the mobility [19]. Remarkably enough, this huge drop in mobility may not only be attributed to oxygen vacancies but also to non-idealities, such as series resistance, in the contact-semiconductor interface. When evaluating carrier mobility from $\left(I_{D}\right)^{1 / 2}$ curves, ideal series resistance at the drain and source contacts have been considered. Higher series resistance will reflect a lower slope in $\left(I_{D}\right)^{1 / 2}$ which can lead to an apparent decrease in the channel mobility [20].

Oxygen vacancies can be reduced by thermal annealing in air atmosphere and would reduce the $n$-type character of the semiconductor [14]. According to reference [5], the oxygen vacancy density is much higher at the surface than in the bulk. When the TFT channel is depleted (i.e. in subthreshold regime) conductivity between drain and source becomes determined by shallow carriers. Therefore, thermal annealing under oxygen ambient reduces the off-current $\left(I_{o f f}\right)$ as depicted in figure 3, which is desirable for a good TFT performance. The drain current in a TFT, which corresponds to the gate-induced current path, takes place mainly near the oxide-semiconductor interface [16]. The observed improvement in mobility for thermally treated TFTs (figure 5) could be also due to a reduction of oxygen vacancies in the deepest part of the semiconductor. Although any annealing process is more effective towards the surface of the layer, the annealing performed in this work has demonstrated to be effective along the whole thickness.

Figure 7 depicts the measured density-of-states of three ZITO layers with different Zn content. All the layers were thermally annealed. The common peak at $0.3 \mathrm{eV}$ is in good agreement to the activation energy of one of the main defects in polycrystalline $\mathrm{ZnO}$ films calculated by Auret et al. [21]. These defects were proposed to be due to oxygen vacancies or zinc interstitials [22]. According to previous measurements with XPS [5], O-vacancy defect density rises in tandem with the $\mathrm{Zn}$ concentration indicating that the observed intensity increase of this peak in figure 7 would correspond to the mentioned oxygen vacancies rather than zinc interstitials. It is also noticeable the remarkable peak at $0.14 \mathrm{eV}$ which is only present in samples with $36 \%$ of $\mathrm{Zn}$ content. This peak was also reported [23] as the activation energy for a shallow-level defect in $\mathrm{ZnO}$ films.

\section{Conclusions}

ZITO films with varying Zn content were investigated by means of ZITO-TFTs with bottom gate-top contact structure. The extracted values for the threshold voltage showed a shift to more positive values as the $\mathrm{Zn}$ content increased, indicating a reduction in the free charge carrier density. We have also found a huge drop in field-effect mobility values probably due 
to an increase in oxygen vacancy concentration causing a higher coulomb scattering of free electrons.

On the other hand, effects of thermal annealing in air atmosphere at $300{ }^{\circ} \mathrm{C}$ were studied. This process demonstrated an improvement in transport characteristics resulting in an increase up to 4 orders of magnitude in mobility values for layers with a high $\mathrm{Zn}$ content. Previously published works established a more $n$-doped surface of the layers as $\mathrm{Zn}$ content increased, which was observed in this work as higher $I_{\text {off }}$ currents. After annealing, all the samples showed a significant reduction of the $I_{\text {off }}$ current. All these mentioned facts led us to conclude that the thermal annealing had the expected effect throughout the whole thickness of the layers, since the mobility is determined by the current near the semiconductor-insulator interface but $I_{\text {off }}$ is dominated by shallow conductivity.

Acknowledgments

This work has been supported by the Spanish government under programs CSD2007-07 from the Consolider HOPE and through the project TEC2011-27859-C02-01 and AMIC (ENE2010-21384-C4-03). It has been also partially supported by the European Community's 7th Framework Programme under grant agreement 227127. We also thank financial collaboration from XaRMAE of the Generalitat de Catalunya.

\section{References}

[1] D.S. Ginley, H. Osono, D.C. Paine, Handbook of Transparent Conductors, ed., Springer, New York, 2011.

[2] A. Facchetti, T. J. Marks, Transparent Electronics: From Synthesis to Applications, ed., John Wiley \& Sons, Chichester, 2010.

[3] D.-S. Liu, Ch-H. Lin, B-W. Huang, Ch-Ch Wu, Jpn. J. Appl. Phys. 45 (2006) 3526-3530.

[4] D.Y. Lee, J.R. Lee, G.H. Lee, P.K. Song, Surf. Coat. Technol. 202 (22/23) (2008) 57185723.

[5] P. Carreras, S. Gutmann, A. Antony, J. Bertomeu, R. Schlaf, J. Appl. Phys. 110 (2011) 073711.

[6] D. B. Buchholz, J. Liu, T. J. Marks, M. Zhang, R. P. H. Chang, Appl. Mater Interfaces 1(10) (2009) 2147-2153.

[7] M. S. Grover, P. A. Hersh, H. Q. Chiang, E. S. Kettenring, J. F. Wager, D. A. Keszler, J. Phys. D: Appl. Phys. 40 (2007) 1335-1339.

[8] J. Liu, D.B. Buchholz, J.W. Hennek, R.P. Chang, A. Facchetti, T.J. Marks, J. Am. Chem. Soc. 132(34) (2010) 11934-11942.

[9] M. K. Ryu, S. Yang, S.-H. K. Park, C.-S. Hwang, J. K. Jeong, Appl. Phys. Lett. 95 (2009) 173508.

[10] M. K. Ryu, S. Yang, S.-H. K. Park, C.-S. Hwang, J. K. Jeong, Appl. Phys. Lett. 95, (2009) 072104.

[11] J. Puigdollers, A. Marsal, S. Cheylan, C. Voz, R. Alcubilla, Org. Electron. 11 (2010) $1333-1337$.

[12] P. Carreras, A. Antony, R. Roldán, O. Nos, P.A. Frigeri, J.M. Asensi, J. Bertomeu, Phys. Status Solidi C 7 (2010) 953-956.

[13] W. Göpel and U. Lampe, Phys. Rev. B 22(12) (1980) 6447-6462.

[14] K. Remashan, D.-K. Hwang, S.-J. Park, J.-H. Jang, Jpn. J. Appl. Phys. 47(4) (2008) 2848-2853.

[15] P. Carreras, A. Antony, F. Rojas, J. Bertomeu, Thin Solid Films 520 (2011) 1223-1227. 
[16] D. Hong, G. Yerubandi, H.Q. Chiang, M.C. Spiegelberg, J.F. Wager, Crit. Rev. Solid State Mater. Sci. 33-2 (2008) 101-132.

[17] T. Globus, H.C. Slade, M.S. Shur, M. Hack, in: M. Hack, A. Madan, A. Matsuda, M. Powell, E.A. Schiff (Eds), Amorphous Silicon Technology, San Francisco, U.S.A. April 1994, Materials Research Society Symposium Proceedings 336 (1994) 883-887.

[18] S.M. Sze, Physics of Semiconductor Devices, second ed., John Wiley \& Sons, New York, 1981.

[19] J. Huang, U.R. Krishna, M. Lemberger, M.P.M. Jank, H. Ryssel, L. Frey, 10th IEEE International Conference on Solid-State and Integrated Circuit Technology (ICSICT) (2010) $1548-1550$.

[20] H.Q. Chiang, Ph.D. Thesis, Oregon State University, USA 2003.

[21] F.D. Auret, S.A. Goodman, M.J. Legodi, W.E. Meyer, D.C. Look, Appl. Phys. Lett. 80 (2002) 1340-1342.

[22] D.M. Hofmann, D. Pfisterer, J. Sann, B.K. Meyer, R.Tena-Zaera, V. Muñoz-Sanjose, T. Frank, G. Pensl, Applied Phys. A 88 (2007) 147-151.

[23] H.L. Tuller, J. Electroceram. 4 (1999) 33-40. 
Figure 1. Schematic cross-section of thin film transistors.

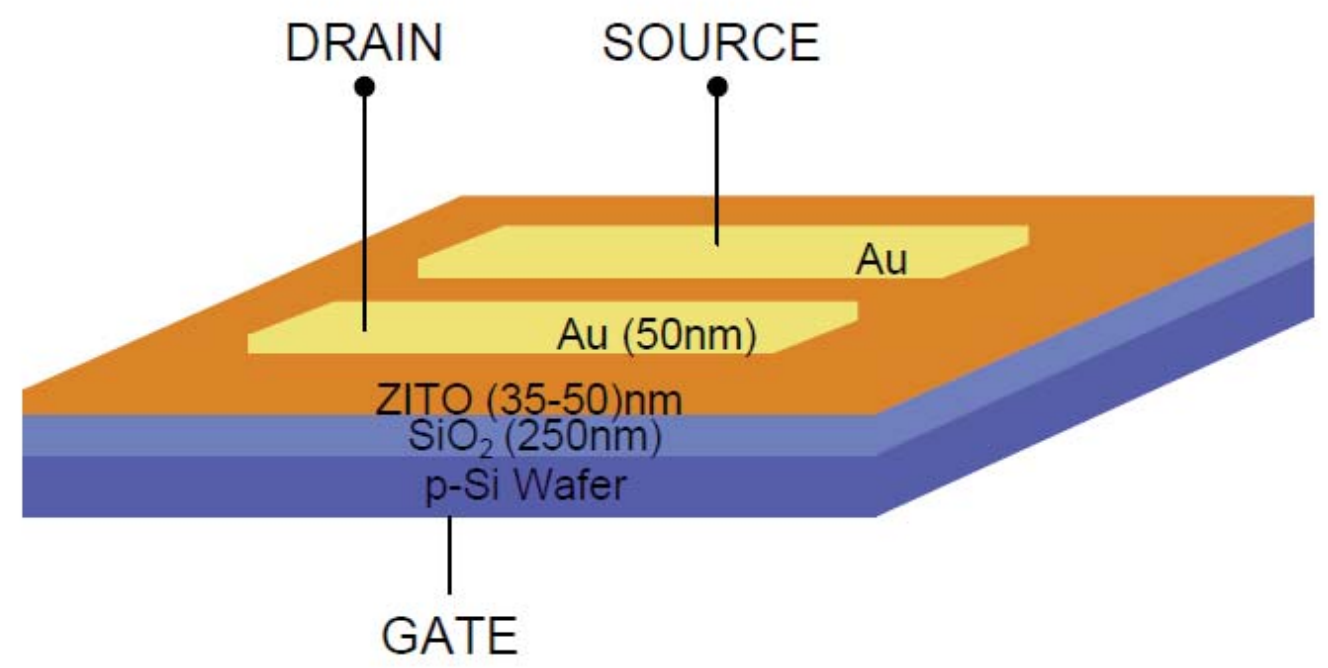

Figure 2. IDS $-V_{D S}$ characteristics for a ZITO50-channel TFT with and without annealing treatment. [ $V_{G S}=0$ up to $20 \mathrm{~V}$ in $4 \mathrm{~V}$ increments].
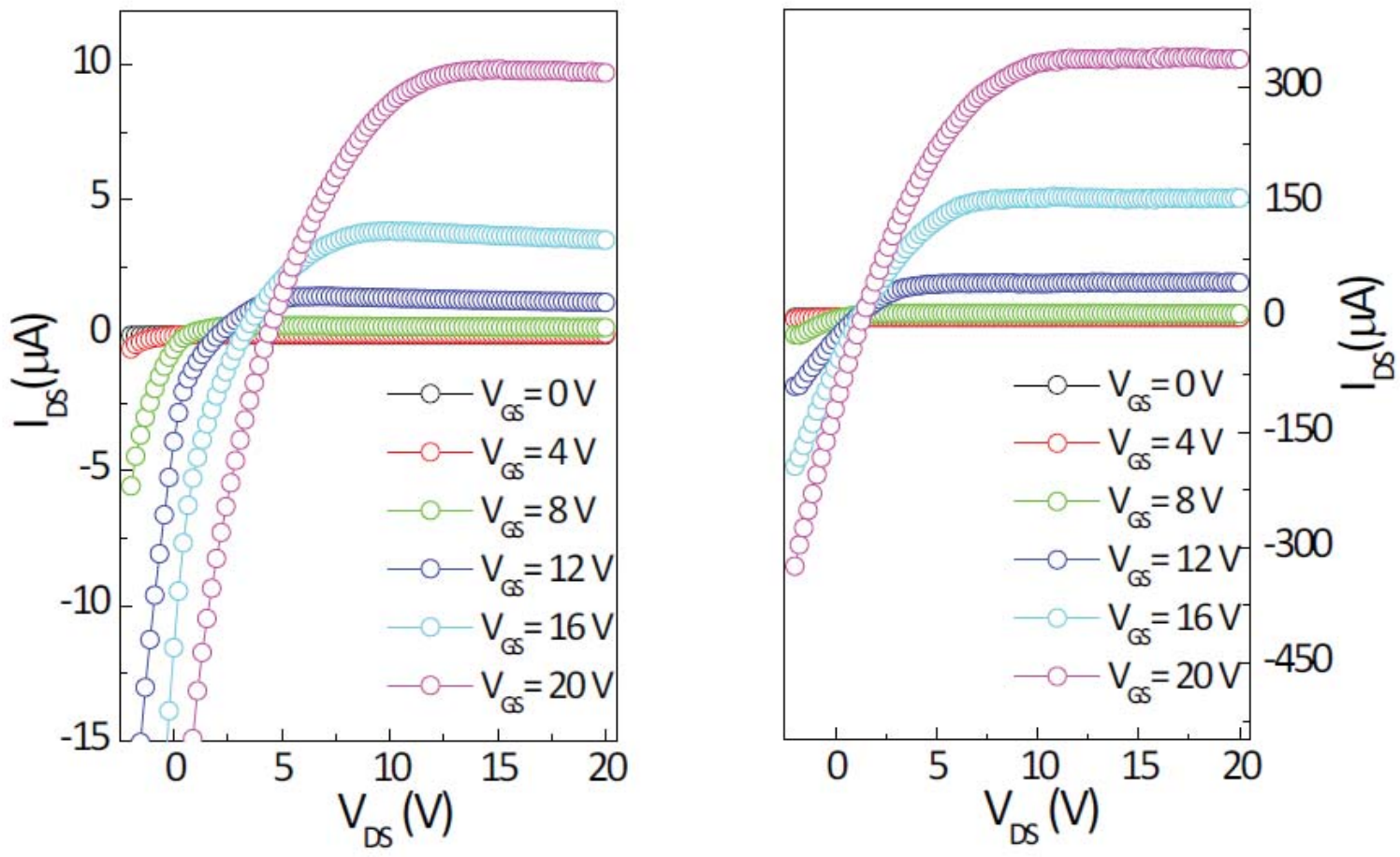
Figure 3. $\log (I D S)-V_{G S}$ characteristics for ZITO100-channel TFTs with a fixed $V_{D S}=20 \mathrm{~V}$. Red curve corresponds to a film with annealing treatment and black curve without annealing.

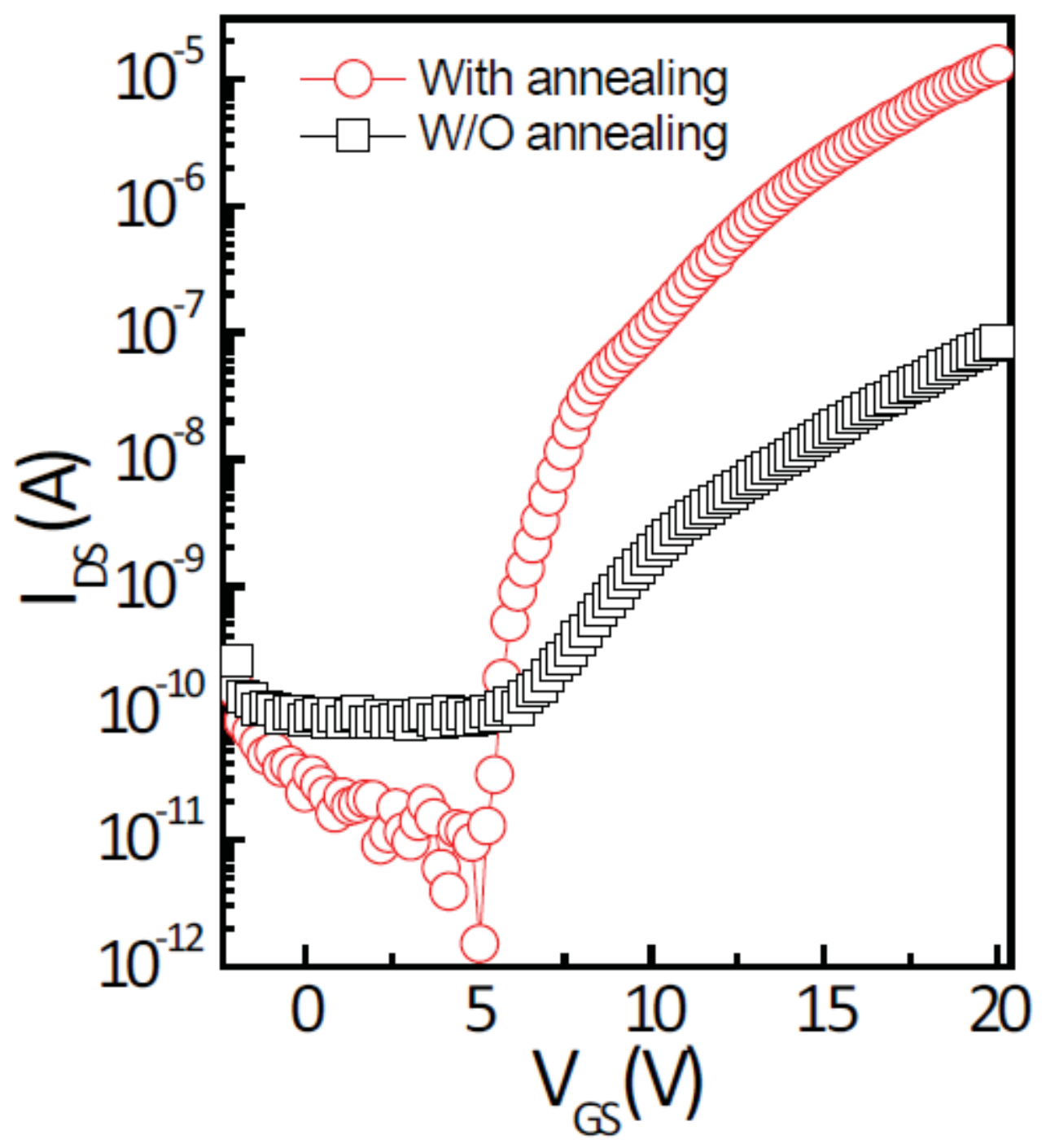


Figure 4. $(I D S)_{1 / 2}-V_{G S}$ with $V_{D S}=20 \mathrm{~V}$ for ZITO-channel TFTs with all studied Zn contents. Right plot corresponds to films with annealing treatment and left plot without annealing.
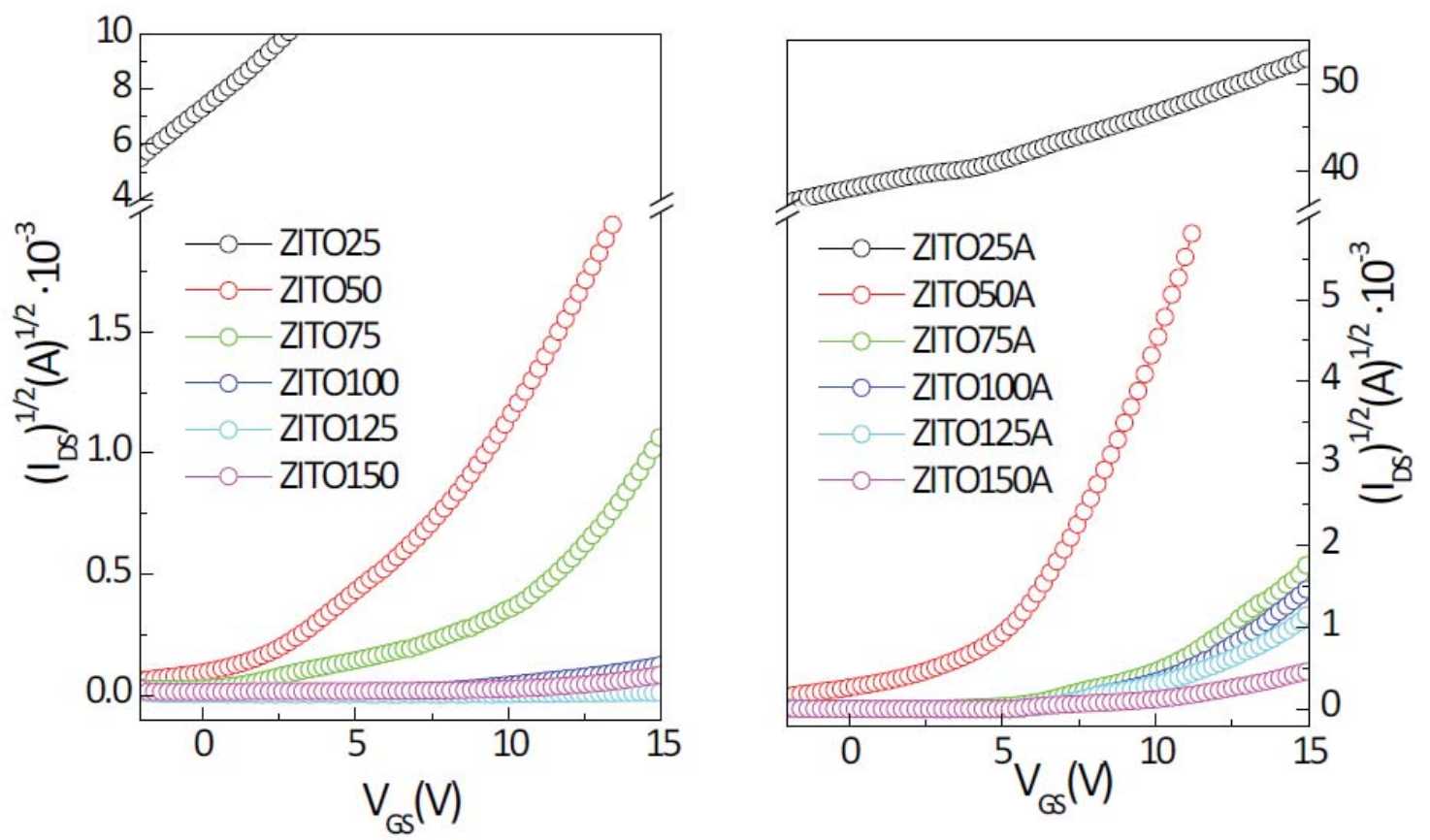

Figure 5. Field-effect mobility (left side) and threshold voltage (right side) as function of $\mathrm{Zn}$ concentration for temperature annealed films (red plot) and without annealing (black plot).
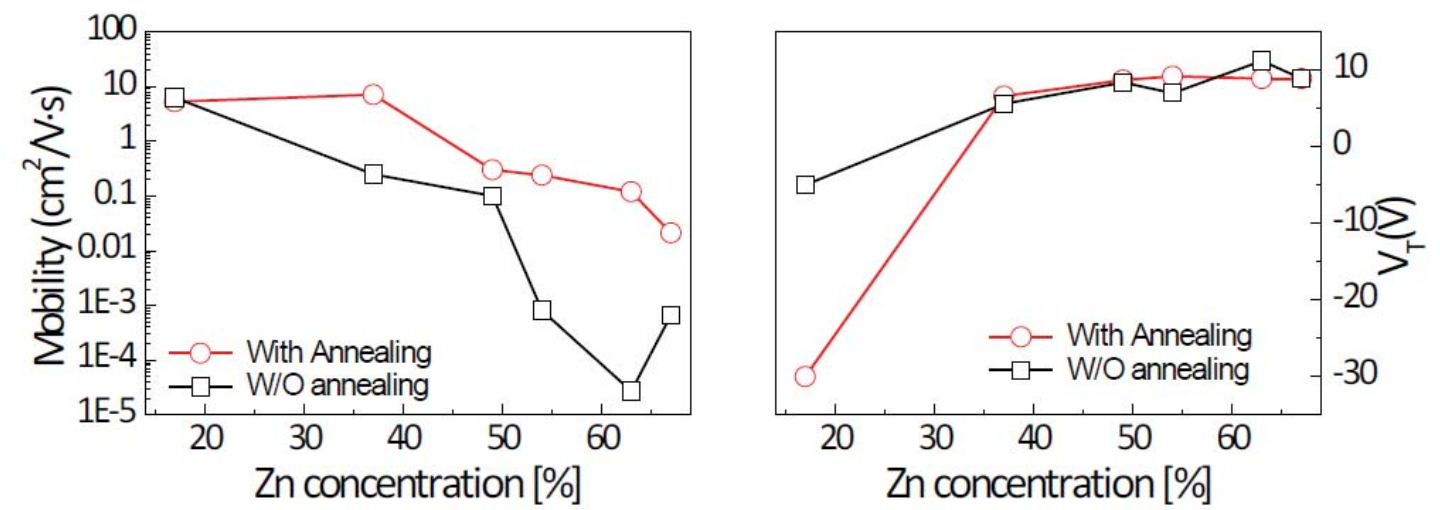

Figure 6. Transfer characteristics of ZITO100-channel TFT with annealing treatment measured at different temperatures $(300-370 \mathrm{~K})$ with $V_{D S}=20 \mathrm{~V}$. The inset shows the 
activation energy of the channel conductance as function of gate voltage.

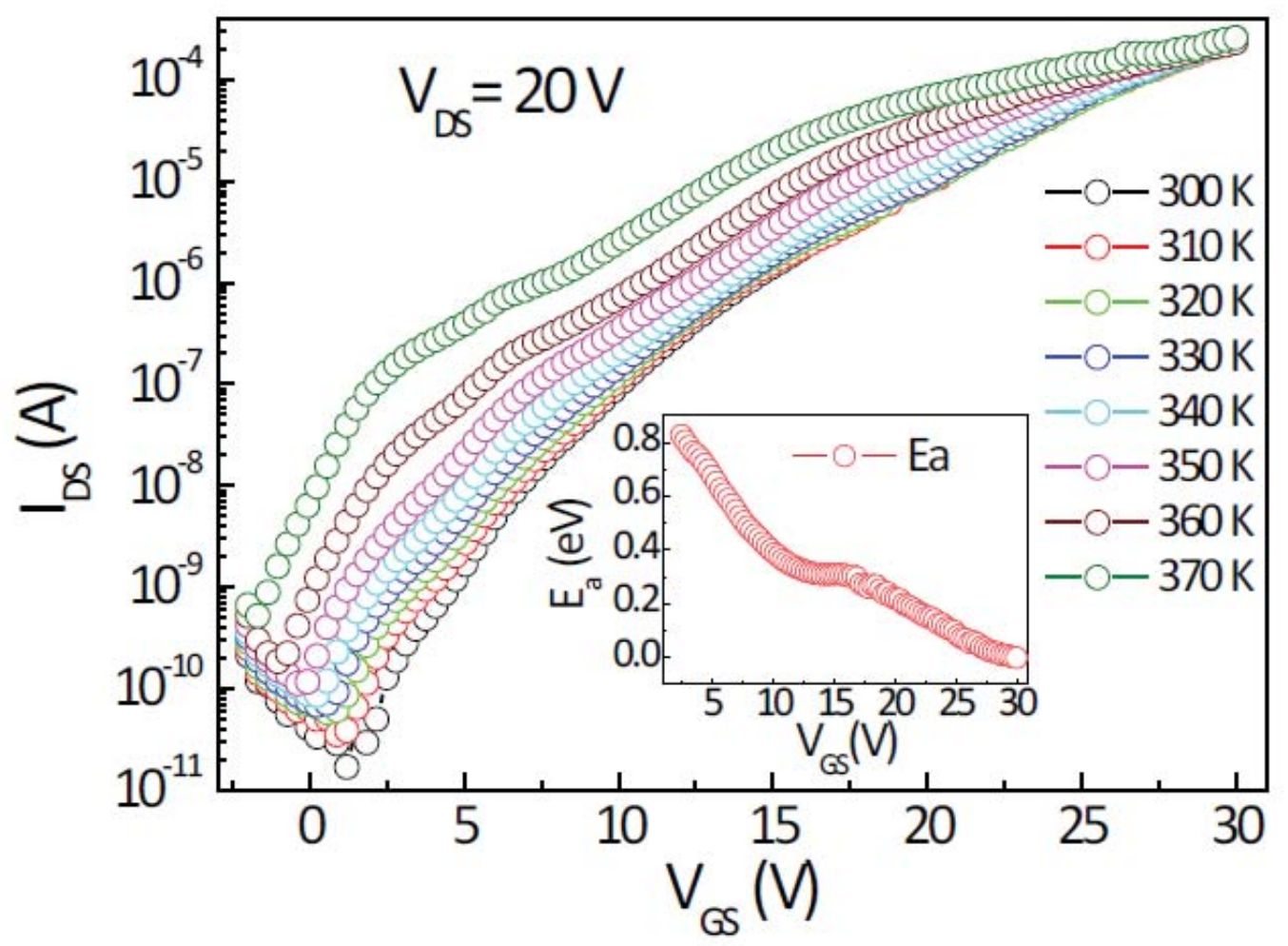

Figure 7. Calculated DOS for 3 different ZITO films with annealing treatment. 


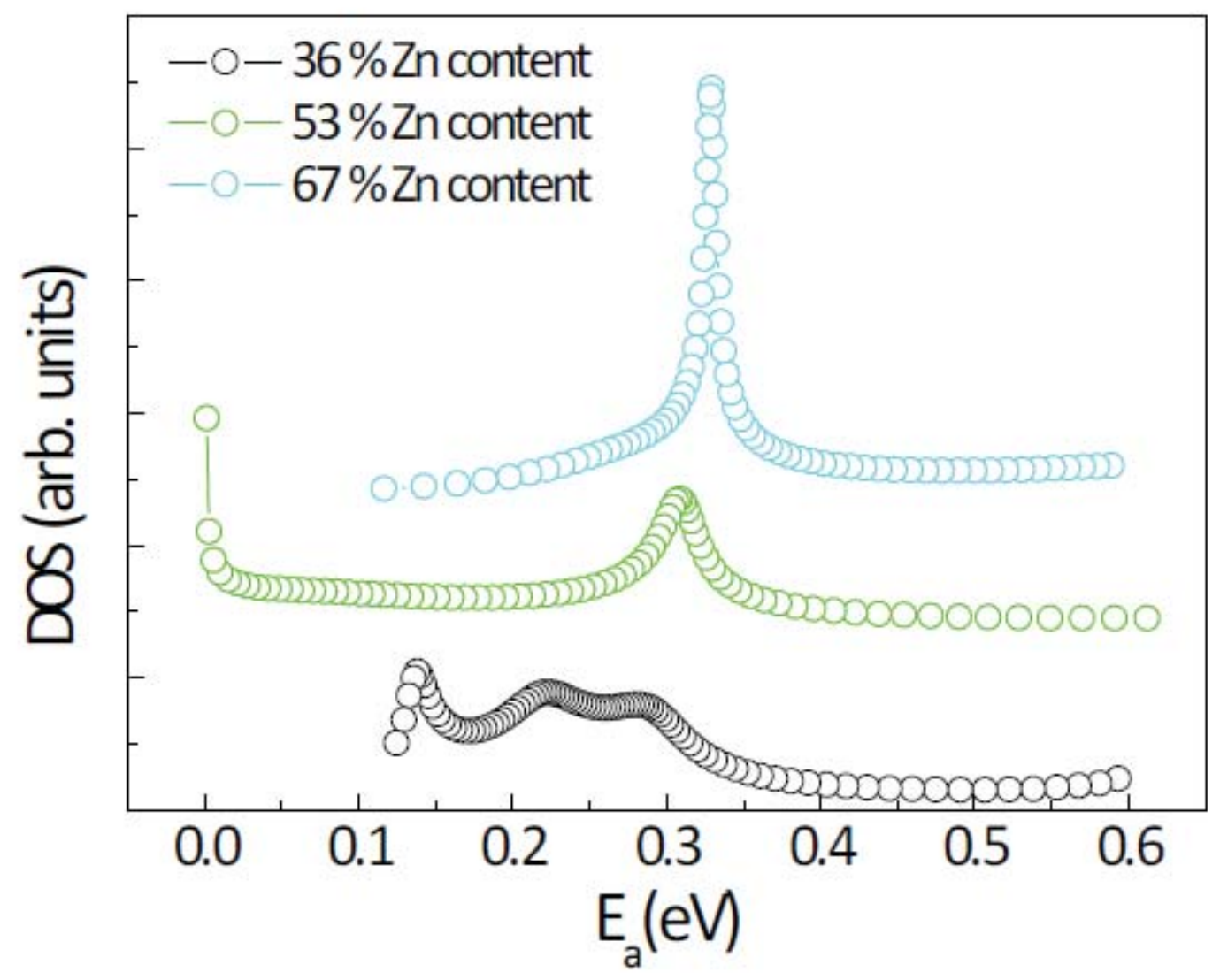

Table 1. Deposition power, cation content ratio and four point measured resistivity of the different samples.

\begin{tabular}{|l|l|l|l|l|l|l|}
\hline $\begin{array}{l}\text { Sample } \\
\text { Name } \\
\text { thickness } \\
\text { nm })\end{array}$ & $\begin{array}{l}\text { ZnO rf } \\
\text { power }(\mathrm{W})\end{array}$ & $\begin{array}{l}\text { ITO rf } \\
\text { power }(\mathrm{W})\end{array}$ & $\begin{array}{l}\text { Zn content } \\
\text { ratio }(\%)\end{array}$ & $\begin{array}{l}\text { In content } \\
\text { ratio }(\%)\end{array}$ & $\begin{array}{l}\text { Sn content } \\
\text { ratio }(\%)\end{array}$ & $\rho(\Omega \cdot \mathrm{cm})$ \\
\hline $\begin{array}{l}\text { ZITO25 } \\
(35)\end{array}$ & 25 & 50 & 17.1 & 75.5 & 7.5 & $9.56 \mathrm{E}-03$ \\
\hline $\begin{array}{l}\text { ZITO50 } \\
(40)\end{array}$ & 50 & 50 & 36.6 & 57.6 & 5.8 & $1.95 \mathrm{E}-02$ \\
\hline $\begin{array}{l}\text { ZITO75 } \\
(45)\end{array}$ & 75 & 50 & 48.5 & 46.7 & 4.8 & $3.02 \mathrm{E}-02$ \\
\hline $\begin{array}{l}\text { ZITO100 } \\
(40)\end{array}$ & 100 & 50 & 53.8 & 41.8 & 4.4 & $2.64 \mathrm{E}-02$ \\
\hline $\begin{array}{l}\text { ZITO125 } \\
(50)\end{array}$ & 125 & 50 & 62.8 & 34.5 & 2.7 & $7.65 \mathrm{E}-02$ \\
\hline $\begin{array}{l}\text { ZITO150 } \\
(45)\end{array}$ & 150 & 50 & 67.3 & 30.2 & 2.6 & $8.10 \mathrm{E}-02$ \\
\hline
\end{tabular}

